Physica C

24/DFL 4615. - /papers

Editor/s: * R.P. Griessen

Natuurkungig Laboratorium

Vrije Univ., Amsterdam

Physica Scripta

12/papers/DM 1500.-

- submission to specialist Editors

Honorary Editors:

L. Hulthén, B. Edlén

Plasma Physics \& Controlled Fusion

14/Fre, Ger, Rus/reviews, papers, letters instn DM 2033.- ; person DM 1070 .

TEX, LATEX, AMSTEX

Deputy Editor/s: R.J. Hastie

Culham Lab., Abingdon, UK

Reports on Progress in Physics

12/£350.-/reviews

TEX source code

Honorary Editor: M. Hart, Univ.

Manchester

Semiconductor Science \& Technology

12/£258.-/papers, letters

Deputy Editor: J.W. Orton

Philips Res. Labs., Redhill, UK

Zeitschrift für Physik A

12/Ger/DM 1680.-/papers

Editor-in-Chief: ' B. Povh

MPI f. Kernphysik, Heidelberg

Zeitschrift für Physik B

12/Ger/DM 2196.-/papers

Editor/s-in-Chief: * H. Horner, Heidelberg

Zeitschrift für Physik $C$

16/Ger/DM 2996.-/papers

Editor-in-Chief: * G. Kramer

Inst. f. Theoretische Physik

Univ., Hamburg

Zeitschrift für Physik D

16/Ger/DM 1936, -/papers

Editor-in-Chief: * I.V. Herte

Fak. f. Physik, Univ., Freiburg i. Br.
Desk Editor: * P.J. Hoff

Elsevier Science Publishers B.V.

Executive Editor: * A. Bárány

The Royal Swedish

Academy of Sciences

Box 50005

S-104 05 Stockholm

- Managing Editor

OP Publishing Ltd \&

Pergammon Press

In assocn. with EPS Plasma

Physics Division

Managing Editor: * R.J. Palmer IOP Publishing Ltd.

Managing Editor: • R.J. Palmer IOP Publishing Ltd.

Springer-Verlag GmbH \& Co. KG

Springer-Verlag GmbH \& Co. KG

Springer-Verlag GmbH \& Co. KG

Managing Editor: - I.V. Hertel Springer-Verlag GmbH \& Co. KG

address for submissions issues p.a./languages/contents prices

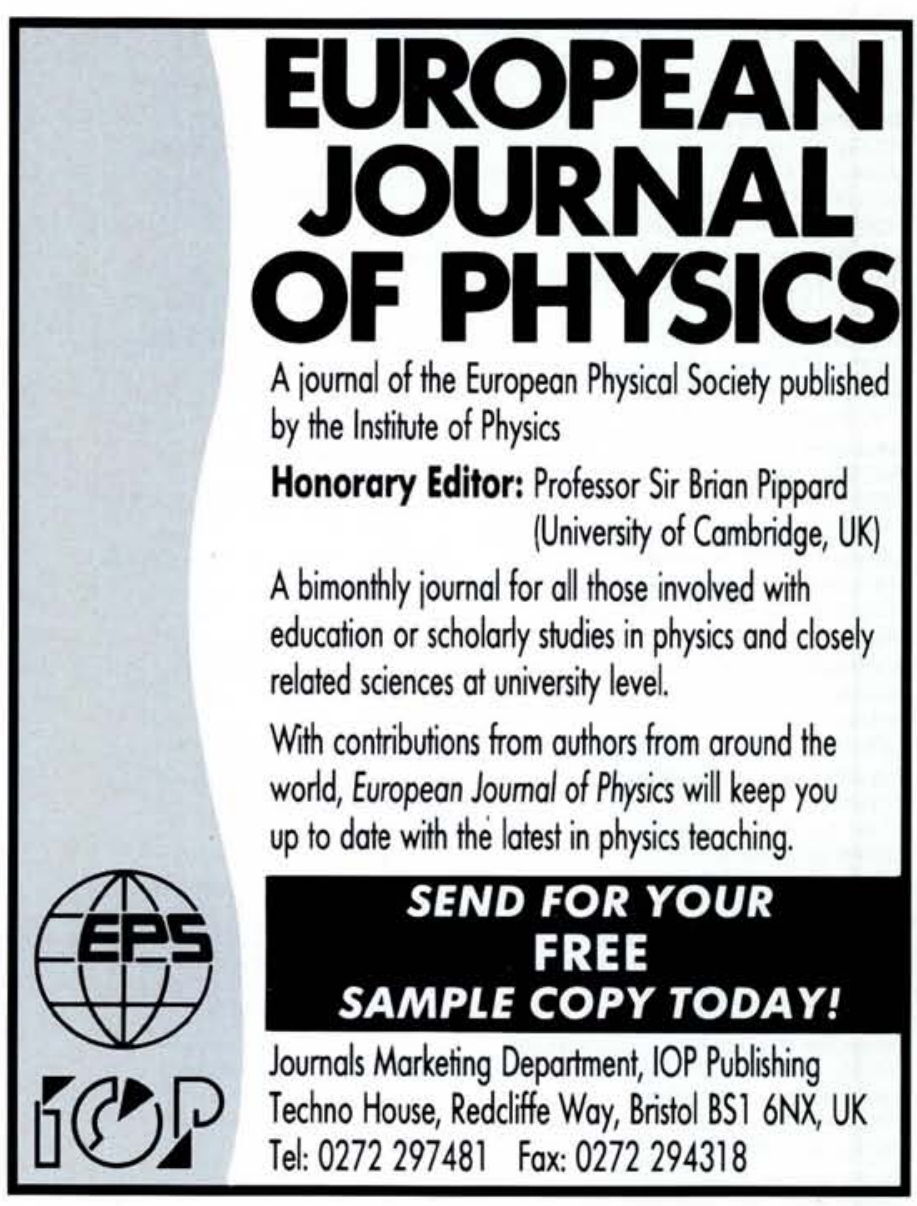

\section{Rare Decays and Fundamental Processes}

\section{4th EUROPHYSICS CONFERENCE ON NUCLEAR PHYSICS}

\author{
22-26 October 1990, Bratislava, Czechoslovakia
}

The fourteenth in the series of Europhysics Conferences organized by the Board of the Nuclear Physics Division of EPS, Bratislava University and the Union of Slovak Mathematicians and Physicists was held on 22-26 October 1990 in Bratislava, Czechoslovakia. It was the first time that an EPS Nuclear Physics Divisional Conference took place in a central/east European country.

The conference was devoted to Rare Decays and Fundamental Processes. The aim was to provide a forum for presenting and discussing the most recent data becoming available from a variety of specific experimental probes, as well as to discuss recent theoretical results stimulating progress in the field. These topics were chosen not only because of a traditional research orientation of the host, Bratislava University, but also to show the potential for non-accelerator techniques for solving important problems of contemporary nuclear and subnuclear physics.

The conference was attended by 210 participants representing 27 countries from all over the world, and 3 internatio- nal organizations. The participants pre56 papers in poster sessions. All exciting problems in rare nuclear phenomena were covered, e.g. rare decays of particles and nuclei, double beta decay, neutrino mass, solar neutrinos, dark matter, proton decay, $\mathrm{N}-\mathrm{N}$ oscillations, rare heavy ions processes, underground laboratories, accelerator mass spectrometry, low temperature detectors, cosmogenic radioactivity, etc.

The opening ceremony took place in the University Aula with about 400 participants and Bratislava physicists, where Nobel laureate R.L. Mössbauer presented his honorary opening lecture on a new generation of radiation detectors based on low temperature physics phenomena. Promising results obtained until now (also described by other speakers) showed that a revolution in radiation detectors is ahead of us that may considerably influence future research, e.g., the search for dark matter. A. Faessler in his opening lecture discussed with great professionalism the links between the particle and nusented 91 papers in oral sessions and

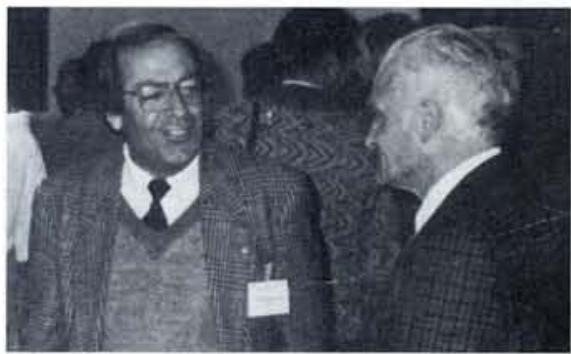

Professor R. Bock (right), Chairman of the International Advisory Committee of the 14th EPS Nuclear Physics Conference, with Professor A. Faessler.

clear physics, using the familiar examples of grand unification and double beta decay.

Among the main hits of the conference belonged new observations of a possible $17 \mathrm{keV}$ neutrino in beta decay (E. Norman, LBL Berkeley; A. Ljubičič, IRB Zagreb). These observations support previous results of Simpson who claimed the existence of a $17 \mathrm{keV}$ heavy neutrino from the beta spectra of ${ }^{3} \mathrm{H}$ and ${ }^{35} \mathrm{~S}$.

Several speakers reported new results of a search for the neutrino mass by measuring the beta-spectrum of tritium. The lowest limits were obtained using free molecular hydrogen $-9.4 \mathrm{eV}$ at $95 \%$ confidence level (T.J. Bowles, LANL Los Alamos). A solid tritium 
source gave the best limit of $13 \mathrm{eV}$ at $95 \%$ confidence level (S. Shibata, INS Tokyo).

New results on two neutrino double beta decays of ${ }^{76} \mathrm{Ge}$ and ${ }^{100} \mathrm{Mo}$ presented by several groups (F.T. Avignone III, USC Columbia; I. Kirpichnikov, MEP Moscow; M.K. Moe, UCI Irvine; H. Ejiri, Osaka University) showed half-lives of $10^{19}-10^{21}$ years which represent the slowest process observed till now in nature.

Several new major experiments are collecting data or they are under preparation. Enriched ${ }^{76} \mathrm{Ge}$ crystals, time projection chambers and cryogenic de- tectors will look for the more exciting neutrinoless double beta decay. The best present limit for the neutrinoless double decay is $2 \times 10^{24}$ years, obtained from ${ }^{76} \mathrm{Ge}$ experiments (D.O. Caldwell, UCB Santa Barbara and F.T. Avignone III, USC Columbia). This constrains the electron neutrino mass to be less than about $2-3 \mathrm{eV}$, if the neutrino is a Majorana particle. Hunting for the neutrinoless double decay is the main programme for the near future. This was pointed out several times during a special workshop on the future of double beta decay experiments. A TPC using a tonne of ${ }^{136} \mathrm{Xe}$ could reach a neutrino mass limit of $10^{-3} \mathrm{eV}$. There has also been considerable progress in double beta decay theory. The theoretical groups understand how the matrix elements required to deduce neutrino mass limits from neutrinoless double beta decay rates can be reliably calculated (A. Faessler, Tübingen University; P. Vogel, Caltech Pasadena; H.V. KlapdorKleingrothaus, MPI Heidelberg). The double beta decay remains a sensitive probe of physics beyond the standard model.

General phenomenological schemes of neutrino mixing were reviewed by S.M. Bilenky. He discussed recent experimental data including LEP measurements of the number of neutrino species. The existing data cannot exclude the possibility that current neutrino fields are superpositions of fields of three light neutrinos and a heavy neutrino. Future direct counting solar neutrino experiments and accelerator experiments including $c-\tau$ factories were considered to be important. Several underground installations for neutrino physics (GALLEX, LVD, MACRO, GALLIUM), a proton decay (SOUDAN 2, IMB, Fréjus) and a monopol search (MACRO) were described and first results were presented.

fessorship in the field. The position will then be announced.

The Solid State Group is a subdivision of the Physics Department, which has about 60 permanent scientific positions. The scientific activities are mainly concentrated on Low Temperature Physics (superconductivity) and Cooperative Phenomena (including phase transitions and disorderly transport processes). The University is primarily looking for a theorist who can stimulate the low temperature activity and establish an advanced programme in quantum condensed matter physics. Applicants in a position to strengthen other activities of the Group will, however, also be considered. The theorist is expected to work in conjunction with experimental activities in the Group, hence the applicant must document experience from collaboration with experimentalists.

The salary is NOK 288011 p.a. after deduction of $2 \%$ to the State pension fund.

The conditions of appointment to Professor include taking part in teaching and examinations according to the curriculum. Changes concerning range of subjects, as well as pensions scheme or age of compulsory retirement, established by law or by the Government, must be accepted without compensation.

Applicants have to send the following documents within one month after the closing date for application to the Faculty of Mathematics and Natural Sciences, Postbox 1032, University of Oslo, Blindern, 0316 Oslo 3, Norway: 5 complete sets of scientific papers, published and unpublished, which are to be taken into consideration by the evaluation, 6 copies of a list including titles of all papers followed by information of where and when they have been printed and 5 copies of the application with possible enclosures. However, scientific papers under completion may be sent on within a period of three months after the closing date, on condition that these papers have been mentioned by the applicant when the other papers were sent.

Shortly after the closing date all applicants will receive further information about the handing in of scientific papers. The procedure for appointment to Professor is based on regulations laid down by the Senate.

If two applicants are assessed as being equivalent, based on their scientific/professional and educational qualifications, a female applicant will be given rank above a male applicant.

The application with curriculum vitae and publication list should be sent to:

The Director of the University of Oslo, The University of Oslo,

Postbox 1072, Blindern, 0316 Oslo 3, Norway.

Closing date for application: November 15th, 1991.

\section{Mathematica}

A System for Doing Mathematics by Computer

A Wolfram Research Inc. product

$\square$ Numerics - Works with numbers of arbitrary magnitude and precision.

Symbolics - Encyclopaedia of mathematical functions and operations used in arithmetic, algebra and analysis.

Procedural, functional and mathematical programming.

$\square$ Graphics - 2D, 3D and animated PostScript graphics.

Text processing - Fully interactive reports and textbooks.

$\square$ Runs on - MS-DOS based computers; Macintosh, Apollo, Hewlett Packard, IBM AIX/RT, MIPS, Silicon Graphics, Sony, Sun, VAX.

Now available in Europe from:

MathSoft Overseas, Inc.

POB 641, 1211 Geneva 3, Switzerland

Tel. ++41 (22) 465260

Fax $++41(22) 465939$ 
Several other rare nuclear processes were discussed, e.g., higher-order processes in nuclear decay (A. Wanda, Torino University), rare effects in gamma-ray internal conversion (O. Dragoun, INP Rež), two-proton radioactivity ( $F$. Pougheon, INP Orsay), etc.

Accelerator mass spectrometry has proved to be a very sensitive technique when applied to half-live measurements $\left({ }^{32} \mathrm{Si},{ }^{41} \mathrm{Ca},{ }^{44} \mathrm{Ti},{ }^{60} \mathrm{Fe},{ }^{126} \mathrm{Sn}\right)$ and searches for hypothetical particles (V. Kutschera, ANL Argonne). Concentration limits for fractionally charged particles (FCP) as low as $10^{-19} \mathrm{FCP} / \mathrm{nu}$ cleon were reported. Non-Paulian atoms and non-Paulian nuclei with three identical particles in the $1 \mathrm{~s}_{1 / 2}$ shell, thus violating the Pauli exclusion principle, were looked for ( $E$. Nolte, TU Munich). The best limit obtained for non-Paulian atoms is $\left[{ }^{20} \tilde{\mathrm{Ne}}\right] /\left[{ }^{20} \mathrm{Ne}\right]<2 \times 10^{-21}$ and for non-Paulian nuclei $\left[{ }^{5} \mathrm{He}\right] /\left[{ }^{5} \mathrm{He}\right]<2$ $\times 10^{-15}$.

Rare heavy ion processes were widely treated from the theoretical as well as experimental points of view. The theory of cluster decay, cold fusion and cold fission has been unified with strong potentialities for predictions of characteristics of nuclei. (A. Sandulescu and D.N. Poenaru, IAP Bucharest). New observations of cluster radioactivity of heavy nuclides (S.P. Tretyakova, JINR Dubna) and the fine structure in the energy spectrum of ${ }^{14} \mathrm{C}$ radioactivity (E. Hourani, IPN Orsay) documented a great success in the field from 1984 when Rose and Jones discovered the ${ }^{14} \mathrm{C}$ radioactivity of ${ }^{223} \mathrm{Ra}$. Rare heavy ion reactions close to the Coulomb barrier, e.g., sub-barrier fusion enhancements, nucleon transfer at large internuclear distances and elastic scattering are linked together with the mechanism of channel coupling that is responsible for experimental evidence (A.M. Stefanini, INFN Legnaro).

Rare decays of cosmogenic and antropogenic radionuclides in extraterrestrial and terrestrial samples and their utilization for studying the origin of cosmic rays from supernovae (G.E. Kocharov, FTI Leningrad), characteristics of the sun (P.E. Damon), exposure ages of meteorites (K. Yamakoshi, Tokyo University), global atmospheric mixing ( $\mathrm{K}$. Rozanski, IAEA Vienna) and environmental physics (K.O. Münnich, Heidelberg University) are only a few examples demonstrating the wide range of rare nuclear phenomena.

\section{Other Activities}

Three workshops - High precision ${ }^{14} \mathrm{C}$ measurements (Moderator: A. Long), Future double beta decay experiments
(Moderator: H.V. Klapdor-Kleingrothaus) and Low-level radioactivity in the environment (Moderator: K.O. Münich) enabled a closer discussion of the topics of interest.

A panel discussion - Future accelerators for nuclear physics and technology in Europe - moderated by C. Détraz (Chairman of NuPECC) was devoted to recent activities on the future orientation of nuclear research in central and eastern Europe. Plans for the installation of new accelerator centres were presented - the AUSTRON project for a c- $\tau$ factory or a proton accelerator with a spallation neutron source located in central Europe (M. Regler, UHEP Vien- na), and the Dubna project for a C- $\tau$ factory and heavy ion storage rings (R.Ts. Oganessian and E.A. Pereljshtein).

It is impossible to mention all the interesting contributions in this short report. Readers interested in the conference topics should refer to the Conference Proceedings.

The Conference was a continuation in the series on low radioactivities organized by the Brastilava University (No. 4 - Low Radioactivities '90). As many participants asked to meet again in High Tatras, it is planned to prepare the next conference on low radioactivities within a few years at this location.

P. Povenic, Bratislava University

In the

\section{Faculty of Science of the University of Zurich}

a newly created

\section{Professorship in Environmental Sciences}

is to be filled.

The responsibilities comprise research in the field of environmental sciences as well as the administration of interdisciplinary (interfaculty) coordination. The teaching duties involve active participation in already existing programmes at both undergraduate and postgraduate levels. The successful candidate will be expected to give lectures in German within a reasonable period after appointment.

Candidates should show a successful research record in some field of natural science as well as ability and experience in dealing with environmental problems. They should be willing to direct and coordinate interdisciplinary research projects.

Holders of an advanced degree with proven academic experience are asked to send curriculum vitae, list of publications and a short description of their research interests before September 30th, 1991 to:

Dekan der Philosophischen

Fakultät II der Universität,

Winterthurerstrasse 190, $\mathrm{CH}-8057$ Zurich.

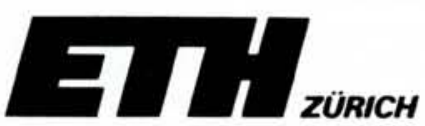

At the Swiss Federal Institute of Technology Zurich (ETH Zurich) applications are invited for a position of

\section{Professor of Theoretical Physics in the field of Condensed Matter Physics}

The duties of this Professor include teaching of undergraduate courses in theoretical physics and advanced courses on special topics as well as the supervision of diploma and doctoral theses. Scientific collaboration with existing experimental and theoretical groups in the Zurich area working in Condensed Matter Physics and Quantum Electronics is encouraged. At present, the research programs of the Theoretical Physics Institute of ETH Zurich are in the areas of Solid State Physics, Particle Physics, Mathematical Physics and Biophysics.

Younger candidates with substantial achievements in research will be preferred. A knowledge of German is not essential, provided a commitment to learn German is made. Applications with curriculum vitae and a list of publications should be sent no later than October 15th, 1991, to the President of ETH Zurich, Prof. Dr. J. Nüesch, ETH-Zentrum, CH-8092 Zurich. In its effort to increase the number of women in academic top positions, the ETHZ specifically invites applications from women. 